\title{
Gerhard Domagk (1895-1964) und die ersten Medikamente gegen Tuberkulose
}

\section{Gerhard Domagk (1895-1964) and the First Medicaments Against Tuberculosis}

Bibliografie

DOI http://dx.doi.org/

$10.1055 / \mathrm{s}-0034-1365458$

Online-Publikation: 8.4.2014

Pneumologie 2014; 68: 394-396

(c) Georg Thieme Verlag KG

Stuttgart · New York

ISSN 0934-8387

Korrespondenzadresse Prof. Dr. Max Hundeiker Immelmannstraße 16 48157 Münster

max@hundeiker.de
In der Erinnerung vieler Mediziner ist Gerhard Domagk vor allem der Mann, der unter der NaziDiktatur den Nobelpreis für die Entwicklung der Sulfonamide nicht annehmen durfte und dafür in Gestapo-Haft geriet. Auffallend wenig bekannt ist aber seine für unsere Zeit folgenreichste Leistung, vielleicht, weil sie wie vieles andere nach dem Ende der Diktatur in andere Hände kam: die Entwicklung der Tuberkulosemittel Conteben (Tb1) und Neoteben (INH). Ihre Bedeutung kann sich kaum vorstellen, wer nicht die Tuberkulose als Volksseuche erlebt hat. Noch um die Mitte des 20. Jahrhunderts war die Lungentuberkulose überall alltäglich. Krankenstationen, ganze Kliniken, Sanatorien waren voll von elend dahinsiechenden hustenden, spuckenden Menschen. In jeder Nachbarschaft gab es „offene“ Tuberkulöse mit oft blutigem Auswurf, von denen man Abstand hielt. Der Gedanke, dass die Luft um Hustende voller Bakterien war, machte den Umgang mit Kranken unheimlich. Befall anderer Organe war weniger auffällig, außer an der Haut: Hauttuberkulose war zwar kaum selbst infektiös („Die Haut hustet nicht!“), führte aber zu furchtbaren Entstellungen. Hautherde konnte man zwar manchmal operativ ganz beseitigen [1,2], aber sonst versuchte man vor allem mit guter Ernährung, Luft, Licht, Sonne, „Abwehrkräfte“ zu stärken. So begann vor 150 Jahren die fast hundertjährige Blütezeit der Lungensanatorien in heilklimatischen Kurorten. Sonnenbestrahlung wurde später durch künstliche UV-Strahlung ergänzt [3]. Zudem hatten in Belgien und Frankreich seit etwa 1942 die Dermatologen Fanielle und Charpy Vitamin D2 erprobt. Das brachte erkennbare Besserungen bei Hauttuberkulose, verlor aber nach wenigen Jahren an Bedeutung, als gegen Mykobakterien wirksame Medikamente entwickelt wurden [4-7]:

In Schweden hatte Jörgen Lehmann seit 1943 mit Para-Aminosalicylsäure (PAS), einer vom Aspirin abgeleiteten Substanz, gearbeitet. Er hatte dabei
1943 in vitro und 1944 im Tierversuch eine antimykobakterielle Wirkung gefunden und PAS noch 1944 auch zunächst topisch an Kranken angewendet. Definitive Heilungen nur durch PAS ohne weitere Maßnahmen waren nicht sicher. Im Januar 1946 erschien in „Lancet“ die erste Publikation darüber [8].

In den USA hatte der Mikrobiologe Selman A. Waksman seit Jahren an Actinomycetales geforscht [9] und 1943 aus Streptomyces grisei das Streptomycin (SM) isoliert. Dessen Hemmwirkung gegen Mykobakterien in vitro bestätigte sich im Tierversuch 1944. Noch 1944 wurde auch schon über mit SM behandelte Kranke berichtet. Hinshaw und Feldman sahen aber ihre eigenen Ergebnisse wegen wechselhafter Verläufe noch als nicht ganz sicher beurteilbar an. Den Durchbruch brachte eine Einzelbeobachtung mit rascher Besserung einer Meningitis tuberculosa bei einem einjährigen Kind [9]. Alles zusammengefasst, waren Publikationen über experimentelle Untersuchungen noch 1944 erschienen, über klinische Anwendungen im September 1945. Erste nachkontrollierte Heilungen kann man für 1947 annehmen. Nun produzierten die Gruppen in den USA Publikationen in rascher Folge und stellten sich fortan mit großem Stolz als die ersten dar, die Erfolge erreicht hatten [11-20].

Jörgen Lehmann in Schweden sah das anders: Seine Tierversuche waren 3 Monate, die ersten klinischen Anwendungen von PAS 8 Monate vor denen mit Streptomycin durchgeführt worden und die Amerikaner mussten das wissen, weil sie inzwischen selbst mit PAS gearbeitet hatten [21 -24]. In Deutschland hatte seit 1932 der Pathologe Gerhard Domagk zusammen mit Chemikern und Medizinern wie Behnisch, Schmidt, Mietzsch, Hackmann und anderen in der Entwicklungsabteilung der Bayer-Werke in Wuppertal-Elberfeld während der Entwicklung der Sulfonamide alle Substanzen auch an Mykobakterien getestet mit anfangs wenig befriedigenden Erfolgen. Aber 1938 
beobachtete er Mykobakterien-Hemmung bei Sulfonamiden mit Thiazol- und 1941 mit Thiodiazol-Gruppen. Behnisch vermutete, dass nicht die Sulfonamid-Struktur für die Wirkung entscheidend war. Deshalb wurden 1941 die ersten Thio-semikarbazone synthetisiert und schon 1942 konnte das 4-acetylaminobenzaldehyd-Thio-semicarbazon, das spätere Tb1/698 (Conteben), in vitro und im Tierversuch geprüft werden. Parallel dazu lief die Entwicklung des Isonikotinsäure-Hydrazid (INH), des späteren Neoteben, das im Prinzip von der Struktur des alten Prontosil abgeleitet war [25-30].

Die klinische Prüfung des TB1 musste unter schwierigsten Bedingungen angefangen werden: Bombenkrieg, zerstörte Verkehrswege, Auslagerung von Kliniken, Zwangs- und Mangelwirtschaft, Parteiwillkür. Sie erschien außerdem zunächst schwierig wegen der schwer zu kontrollierenden wechselhaften Verläufe der Lungentuberkulose. H. Loebell, Direktor der Universitäts-HNO-Klinik Münster machte darauf aufmerksam, dass an der Haut die Entwicklung aller Befunde unmittelbar kontrolliert werden konnte. G. Domagk nahm daraufhin Verbindung zu C. Moncorps auf, dem Direktor der Universitäts-Hautklinik. Die wurde in Personalunion mit der damaligen Lupus-Heilstätte und späteren Fachklinik Hornheide geführt. Deren leitender Oberarzt vor Ort war K. W. Kalkoff. Wie das vor Entwicklung moderner Studiendesigns vielfach Sitte war, nahmen zunächst die beteiligten Ärzte selbst, wie auch schon zuvor Domagk, das Mittel in erhöhten Dosen, um sich seiner Verträglichkeit zu vergewissern. Dann wurde es nach eingehender Aufklärung und Beratung bei freiwilligen, anders nicht behandelbaren Dauerpatienten mit fortgeschrittener Hauttuberkulose eingesetzt. Relativ rasch einsetzende Besserung motivierte die Kranken stark zur Mitarbeit, eine langfristige Nachkontrolle war so relativ einfach zu erreichen [31 -34].

Bei den Flächenbombardements im zweiten Weltkrieg war das Bayer-Gelände in Wuppertal teils anscheinend ausgespart worden. Bei Kriegsende wurden, natürlich nicht nur wegen des TB1 bzw. der Thiosemicarbazone, die Entwicklungslabors von Spezialisten der Siegermächte durchsucht und, was brauchbar schien, wurde mitgenommen. Aber G. Domagk gelang es, selbst Material nach Hornheide zu bringen. Die Substanz wurde teilweise durch Mischen mit dem allen bekannten farbigen Prontosil rubrum „versteckt“ [28], so von den Besatzungs-Inspektoren nicht beachtet und weiter angewendet. Nach einiger Zeit bekam Domagk zu seinem Erstaunen einiges an Unterlagen über Tb1 (nicht aber die Weiterentwicklungen in Richtung INH) zurück. Unter Kontrolle durfte Tb1 hergestellt werden. Die Kontrolleure ließen schließlich die Klinik aus Infektionsangst und Grauen vor sie bedrängenden entstellten Patienten weitgehend in Frieden. So wurden in Hornheide schließlich 1946 mit Auswertung der Akten die ersten definitiven und nachkontrollierten Heilungen Tuberkulosekranker durch Medikamente gesichert. Erst als wieder deutsche Fachzeitschriften erscheinen konnten, wurden sie ab etwa 1947 allgemein bekannt [25-27, 35-38].

C. Hinshaw und W. McDermott wurden daraufhin nach Deutschland geschickt, um aus Frankfurt und Wuppertal wissenschaftliche Unterlagen, Daten von schon über 7000 behandelten Patienten sowie Conteben-Substanz in die USA zu schaffen. McDermott selbst schrieb später darüber [39]: "We were sent over to Germany to investigate reports that, virtually unnoticed by the occupying powers, the Germans had treated some 7000 tuberculous patients with a new synthetic chemical of the thiosemicarbazone series under study by Gerhard Domagk, the discoverer of the first sulphonamide". Im Time Magazine erschien zu dieser Geschichte am 21. November 1969 ein Artikel mit dem Titel "war booty"
(„Kriegsbeute“). In dessen Schlussbemerkungen steht: “Because the drug was developed during the war, the German patents are no good and any US-manufacture can make it". Und in der Tat, die Firmen Squibb und Hoffmann-La Roche arbeiteten in den USA mit der Substanz weiter. „TB I“ wurde amerikanisiert zu „Tibione“. Dabei wurde verbreitet und auch vielfach geglaubt, TB1 sei in den USA entwickelt worden. Im September 1951 war G. Domagk beim 12. Internationalen Kongress für reine und angewandte Chemie in New York. Dort präsentierte H. Fox von Hoffmann-La Roche in Nutley NJ Daten aus der Wuppertaler Beute als vorgebliche eigene Entwicklungen, ohne Domagk auch nur zu erwähnen. Domagk verstand es, mit der Situation diplomatisch umzugehen. Er stellte als „Hydrazon“ das noch wirksamere Isonikotinsäure-Hydrazid (INH, Neoteben) vor. Doch auch die Unterlagen dafür waren Beute gewesen. Möglicherweise waren sie Grundlage für eine sehr rasch noch 1951 in einer wenig bekannten Zeitschrift erscheinende Publikation, die eigene Entwicklungswege und Prioritäten der US-Firmen belegen sollte. Aber die Peinlichkeit dieses Vorgehens wurde dann doch zu groß, zumal sich herausstellte, dass die Synthese der Substanz INH, noch ohne Gedanken an therapeutische Anwendung, schon vor längerer Zeit von Hans Meyer und Josef Mally publiziert worden war. So einigten sich schließlich 1952 die Firmen HoffmannLa Roche und Squibb mit Bayer geschäftlich auf eine Darstellung, nach der sie „zugleich“ die Substanz entwickelt und 1951 in die klinische Prüfung gegeben hätten. Damit konnte Bayer auch selbst wieder diese aus der eigenen Firma stammenden Mittel verkaufen, und so wurden diese auch gleich unter verschiedenen Namen eingeführt (Neoteben, Rimifon, Hydrazid, Tebesium) [29]. Polit-ökonomische Interessen und schreckliche Zeitumstände hatten aus etwa gleichzeitigen, fruchtbaren wissenschaftlichen Arbeiten eine Art Wettrennen mit ungleichen Bedingungen um Priorität und Profit werden lassen. Domagk selbst aber hatte bei alledem, wie aus erhaltenen Erinnerungsnotizen deutlich wird, nur ein Hauptanliegen gehabt: die Bekämpfung der Tuberkulose [29].

In den folgenden Jahren verschwand die Tuberkulose in einem vorher so kaum vorstellbaren Siegeszug der Medizin weitgehend aus Mitteleuropa [40 - 45] - bald auch, bei einer Prävalenz nahe null, aus dem Bewusstsein der meisten Menschen. Andererseits waren von Anfang an bei antituberkulösen Chemotherapeutika und Antibiotika Resistenzentwicklungen der Erreger beobachtet worden [15]. Das führte bald zu jahrzehntelang sich bewährenden planvollen Konzepten einer „Multi-Drug-Therapie“. Aber seit einigen Jahren droht die Tuberkulose aus „Rückzugsgebieten“ in Nachfolgestaaten des sozialistischen Machtblocks, in Armutsländern Afrikas und Asiens und zusammen mit erworbener Immundefizienz zurückzukehren. Neue Medikamente sind in Entwicklung. Aber zuerst muss die Aufmerksamkeit für diese Krankheit wieder zunehmen, damit sie sich nicht wieder in Europa ausbreitet $[46,47]$.

Schon früh war bekannt, dass offenbar die weitaus meisten Menschen mit Tbc infiziert wurden, aber nicht alle Infizierten erkrankten und manche Erkrankten wieder gesundeten. Man wusste, dass jahrzehntelang „verkapselte“ Tbc bei die Immunabwehr beeinträchtigenden Umständen oder Krankheiten wieder „aktiv“ werden konnte. Heute wissen wir zudem, dass nicht nur solche Krankheits-Koinzidenzen, sondern, ähnlich wie bei Lepra, verschiedene angeborene Genpolymorphismen an der unterschiedlichen Widerstands- bzw. Erkrankungsfähigkeit verschiedener Menschen nach Infektion mit Mykobakterien beteiligt sind $[48-50]$. 


\section{Literatur}

1 Moncorps C. Chirurgische Behandlung der Hauttuberkulose einschließlich Wiederherstellungschirurgie. Dermatol Wochenschr 1948; 119: $347-348,547-548$

2 Konz B. Carl Moncorps (1896-1952). In: Plewig G, Löser C, eds. Pantheon of Dermatology. New York-Heidelberg-Berlin: Springer; 2013: $757-760$

3 Jesionek A. Natürliche und künstliche Heliotherapie des Lupus. Zeitschrift für Tuberkulose 1916; 25: 1-11

4 Charpy J. Vitamin D2 in der Behandlung der Hauttuberkulose. Z Hautkr 1949; 7: $81-93$

5 Fanielle G. Die massive Vitamin D-Therapie in der Behandlung tuberkulöser Affektionen „langsamer Entwicklung“. Z Hautkr 1949; 7: $121-126$

6 Kalkoff $K$. Die Thiocarbazontherapie der Hauttuberkulose im Vergleich zur hochdosierten D-2-Therapie. Z Hautkr 1949; 7: 131 - 142

7 Langer E, Nobis $W$. Über die Behandlung der Hauttuberkulose mit hohen Dosen Vitamin D2. Z Hautkr 1949; 7: 101 - 109

8 Lehmann J. Para-aminosalicylic acid in the treatment of tuberculosis. Lancet 1946: $15-16$

9 Waksman SA, Henrici AT. The Nomenclature and Classification of the Actinomycetes. J Bacteriol 1943; 46: 337-341

10 Cooke RE, Dunphy DL, Blake FG. Streptomycin in tuberculous meningitis. A report of its use in an one-year-old infant. Yale J Biol Med 1946; 18: $21-226$

11 Hinshaw HC, Feldman WH, Pfuetzer KH. Treatment of tuberculosis with streptomycin: A summary of observations on one hundred cases. J Am Med Assoc 1946; 132: 778 - 782

12 Hinshaw HC, Feldman WH. Streptomycin; a summary of clinical and experimental observations. J Pediatr 1946; 28: 269-274

13 Hinshaw HC, Pyle MM, Feldman WH. Streptomycin in tuberculosis. Am J Med 1947; 2: 429-435

14 Feldman WH, Karlson AG, Hinshaw HC. Streptomycin in experimental tuberculosis. The effects in guinea pigs following infection in intravenous inoculation. Am Rev Tuberc 1947; 56: 346-359

15 Feldman WH, Karlson AG, Hinshaw HC. Streptomycin-resistant tubercle bacilli, effect of resistance on therapeutic results. A, J Pathol 1947; 23: 874

16 Feldman WH. Streptomycin: Some historical aspects of its development as a chemotherapeutic agent in tuberculosis. Am Rev Tuberc 1954; 69: 859-868

17 Waksman SA. Tenth anniversary of the discovery of streptomycin, the first chemotherapeutic agent found to be effective against tuberculosis in humans. Am Rev Tuberc 1954; 70: 1-8

18 Hinshaw HC. Historical notes on earliest use of streptomycin in clinical tuberculosis. Am Rev Tuberc 1955; 70: 9-14

19 Pfuetze KH, Pyle MM, Hinshaw HC et al. The first clinical trial of streptomycin in human tuberculosis. Am Rev Tuberc 1955; 71: 752 - 754

20 Waksman SA. My life with the microbes. London: Robert Hale; 1958

21 Feldman WH, Karlson AG, Hinshaw HC. Para-aminosalicylic acid in experimental tuberculosis in guinea pigs. Proc Staff Meet Mayo Clin 1947: $22: 473-479$

22 Lehmann J. The treatment of tuberculosis in Sweden with para-aminosalicylic acid; a review. Dis Chest 1949; 16: 684-703

23 Lehmann J. A contribution to the debate on treatment of tuberculosis with streptomycin Acta Tuberc Scand Suppl.. 1950; 26: 108-211

24 Lehmann J. Twenty years afterward. Historical notes on the discovery of the antituberculosis effect of para-aminosalicylic acid (PAS) and the first clinical trials. Am Rev Resp Dis 1964; 90: 953-956

25 Domagk G, Behnisch R, Schmidt HS et al. Über eine neue gegen Tuberkelbazillen in vitro wirksame Verbindungsklasse. Naturwissenschaften 1946; 33: 315

26 Domagk G. Investigations on the antituberculous activity of the thiosemicarbazones in vitro and in vivo. Am Rev Tuberc 1950; 61: 8-19
27 Domagk G, Offe HA, Siefken W. Ein weiterer Beitrag zur experimentellen Chemotherapie der Tuberkulose (Neoteben). Dtsch Med Wochenschr 1952; 77: $573-578$

28 Kalkoff KW. Erinnerungen (unveröffentlichtes Manuskript 1938-1945) und persönliche Mitteilungen.

29 Grundmann E. Gerhard Domagk. Der erste Sieger über die Infektionskrankheiten. Worte-Werke-Utopien. Bd. 13. Münster-Hamburg-London: LIT Verlag; 2001

30 Ehring F. Der Lupus vulgaris- Anfang und Ende in historischer Sicht. Dt Dermatol 1994; 42: 1530-1535

31 Kalkoff KW. Die ersten Tuberkuloseheilungen durch Conteben - 25 Jahre danach. Hautarzt 1973; 24: 546-550

32 Kalkoff KW, Ehring F. Die erste Tuberkuloseheilung mit Chemotherapie: eine Lupuskranke in Hornheide. Pneumologie 1994; 48: 448-452

33 Krutmann J. Die Geschichte der Universitäts-Hautklinik in Münster in Westfalen. Studien zur Geschichte des Krankenhauswesens. Bd. 25. Herzogenrath: Verlag Murken-Altrogge; 1987

34 Moncorps C, Ehring FJ. Prinzipien und Organisation der Prävention de Hauttuberkulose. Tuberkulosearzt 1951; 5: 374-379

35 Moncorps C, Kalkoff KW. Vorläufige Ergebnisse einer Chemotherapie der Hauttuberkulose. Derm Wschr 1947; 119: 359- 362 und Med Klinik 1947; 42: 812-816

36 Böhm H. Der aktuelle Stand der Forschung über neue Chemotherapeutica gegen die Tuberkulose. Dtsch Med Wochenschr 1946; 71: 121 125

37 Joel G. Erfahrungen und Ergebnisse bei der Behandlung der Hauttuberkulose mit TB I/698/E. Z Hautkr 1949; 7: 94 - 101

38 Wagner G. Die Behandlung der Tuberkulose - speziell der Hauttuberkulose - mit dem neuen deutschen Chemotherapeuticum Conteben (TbI 698). Acta Derm Venereol 1951; 31: 482 - 484

39 McDermott W. The story of INH. J Infect Dis 1969; 119: 678 - 683

40 Meyer C. Entwicklung der Tuberkulose in Berlin. Beitr Klin Tuberk Spezif Tuberkuloseforsch 1951; 105: 408-428

41 Gehrels PE, Kalkoff KW. Hauttuberkulose. In: Hein J, Kleinschmidt $\mathrm{H}$ Uehlinger E, Hrsg. Handbuch der Tuberkulose, Bd. 4. Stuttgart: Thieme; 1964: $595-726$

42 Ehring F. Die gegenwärtige Epidemiologie und Bakteriologie der Hauttuberkulose in der Bundesrepublik Deutschland. In Jadassohn W, Schirren CG (eds.) XIII Congressus internationalis Dermatologiae, München, 31.7. - 5.8. 1967, Vol. II.1308-1312

43 Orfanos C. Tuberkulose der Haut. In Gottron HA, Schönfeld W, Hrsg. Dermatologie und Venerologie. Ergänzungs-und Registerband. Stuttgart: Thieme; 1970: 262 - 328

44 Hundeiker M. Die Fachklinik Hornheide und ihr Trägerverein 1932 1992: von der Lupusbekämpfung zur Hautkrebsbekämpfung. In: (Festschrift) Erster Direktor Dr. med. hc. Wilhelm Riehemann zum Abschied. Münster: LVA; 1992: 219-229

45 Daniel A. Abschied von der Tuberkulose. Eine Dokumentation der Bekämpfung und Behandlung der Tuberkulose durch die LVA. Münster: LVA; 1989

46 Loddenkemper R, Hauer B. Resistente Tuberkulose - Große Herausforderung durch eine Weltepidemie. Dtsch Arztebl 2010; 107: 10 - 19

47 Hauer B, Fiebig L, Brodhun B et al. Aktuelle Epidemiologie der Tuberkulose weltweit, in Europa und in Deutschland. Pneumologe 2014; 11: $10-20$

48 Hundeiker M, Perusquia-Ortiz AM, Bassukas ID. El futuro de la lepra: Ilusiones y realidad. Dermatol Rev Mex 2013; 57: 491 - 495

49 Yang Y, Li X, Cui W et al. Potential association of pulmonary tuberculosis with genetic polymorphisms of toll-like receptor 9 and interferongamma in a Chinese population. BMC Infect Dis 2013; 13: 511

50 Wang C, Chen ZL, Pan ZF et al. NOD2 Polymorphisms and Pulmonary Tuberculosis Susceptibility: A Systematic Review and Meta-Analysis. Int J Biol Sci 2013; 10: 103-108 